\title{
Network Characteristics Enabling Efficient Coordination: A Simulation Study
}

\author{
Abhimanyu Khan ${ }^{1}$ - Ronald Peeters ${ }^{2}$. \\ Frank Thuijsman ${ }^{3}$. Philippe Uyttendaele ${ }^{3}$
}

Published online: 25 September 2015

(C) The Author(s) 2015. This article is published with open access at Springerlink.com

\begin{abstract}
Using data generated by extensive simulations of a process where individuals interact globally in a coordination game and iteratively imitate the action of the most successful individual in their local neighbourhood, we study the characteristics of the observation network and distribution of initial choices that facilitate (a speedy) convergence to efficient coordination. Knowing these characteristics is crucial when intervening in social network structures with the intention to nudge society to a socially preferred outcome. The most important factor appears to be the share of individuals that are initially seeded with the socially desirable action. Only for a small window of this share, other factors, including the degree distribution in the network and the segregation of individuals using similar actions, have an influence on the dynamic process of achieving efficient coordination. Moreover, networks possessing the properties of a scale-free network are more likely to yield an efficient outcome compared to small-world networks.
\end{abstract}

Keywords Coordination · Local imitation $\cdot$ Global random interaction · Networks

JEL Classification C73

Ronald Peeters

r.peeters@maastrichtuniversity.nl

Abhimanyu Khan

ak933@cam.ac.uk

Frank Thuijsman

f.thuijsman@maastrichtuniversity.nl

Philippe Uyttendaele

phil.uyttendaele@gmail.com

1 Cambridge-INET, University of Cambridge, Sidgwick Ave, CB3 9DD Cambridge, UK

2 Department of Economics, Maastricht University, P.O. Box 616, 6200 MD Maastricht, The Netherlands

3 Department of Knowledge Engineering, Maastricht University, P.O. Box 616, 6200 MD Maastricht, The Netherlands 


\section{Introduction}

Networks - social, economic, institutional, or others - are an important determinant of social and economic behaviour by governing the manner in which information flows amongst individual units. Network characteristics such as the density of links (or connections), the asymmetric influence that certain individual units may bear on the network, and the segregation of individual units in the network have a direct bearing on social and economic outcomes. This paper presents a simulation study based on an evolutionary approach in the context of coordination games (or coordination problems). The aim is to analyse and examine features of a network that determine the standard or convention that a society adopts. Important questions include: Does a society always settle on the better (best) convention (or standard), and what are the network features which facilitate the better (best) coordination outcome?

A coordination game (such as the Stag-Hunt game) is a simple representation of a situation where individuals are better served by coordinating on the same action. An important aspect of coordination games concerns the specific coordination outcome; individuals may coordinate on an outcome that is preferred by all or they may end up coordinating on a relatively undesirable outcome. An archetypical example of coordination games involves technology adoption. When multiple technologies compete, it is often seen that society would benefit by the adoption of any one technology as a standard, as it is more conducive for collaboration amongst individuals. The question is which technology will be adopted? This issue is even more pertinent when one technology is better than the other, but its adoption may be stymied by the absence of a critical mass of users or due to asymmetries in compatibility between standards. Thus, while use of any technology as a standard represents an equilibrium, the particular equilibrium described by adopting the superior technology is better than any other equilibrium where one of the other inferior technologies is adopted as the standard. The determination of the equilibrium that will prevail is a non-trivial question and the process by which individuals obtain information about the current state of the world plays a crucial role. In addition, initial configurations of the individuals' choices (via path dependence) also have a major say. This paper attempts to undertake an exploratory study into the features of networks and the initial configurations that influence the emergent outcome in a stylised environment, taken from Khan [12] and described below. ${ }^{1}$

We consider a finite group of individuals that form a society. Some individuals are linked to each other via social or other ties. These ties or connections permit individuals to exchange information in an undirected way, i.e. information flows in both directions. In addition to this, each individual is randomly matched to another (and not necessarily to someone with whom one is connected) to interact in a strategic setting described by a coordination game. One can interpret this random matching of two individuals as a situation where two individuals, who might not have any information about each other, have to interact with the technology as the interface. With the proliferation of online interactions, individuals are increasingly exposed to interactions that are to a certain extent anonymous, and the assumption of random matching of individuals is intended to capture this feature. Each individual can choose between two actions (e.g. choice of technology), say $P$ and $R$. While it is more profitable to coordinate on the payoff-dominant action $P$ than to coordinate on the less-risky action $R$, in case of mis-coordination it is preferable to choose action $R$.

\footnotetext{
1 The environment described in this paper may also be seen as a study in the broad area of emergence of institutions. There is a wide literature in economics that deals with how institutions emerge endogenously from economic motives. This paper shares a similarity in theme in that we are also interested in what emerges as a result of individuals' incentive to coordinate amongst themselves. In the conclusion of this paper, we briefly remark on how the results of the paper bear on this aspect.
} 
Individuals choose an action, and the payoff for each individual depends on his own action and that of the randomly chosen co-player. After an individual receives the payoff, he exchanges information about his experience (i.e. action used and payoff received) with other individuals with whom he shares a social connection. On having obtained such information from his social links, he imitates the action of the most successful individual in his social circle in the next period, when he will be matched to another randomly chosen individual. The characteristics of the network shape the way in which information exchange takes place, and how individuals choose one action over the other. This situation ensues recurrently, and eventually this process will lead to a situation where the entire population coordinates on one particular action. Our interest lies in the possibility of coordinating on the efficient or preferred equilibrium (all play action $P$ ). The questions that we aim to answer are: (1) What are the important characteristics of the network that facilitate coordinating on the efficient equilibrium?, (2) what is their effect on the time needed to settle on this equilibrium? While the first question addresses the issue of efficiency directly, the second question examines the issue of the time taken to reap the gains of coordinating on the more efficient equilibrium (a delay in achieving efficient coordination may be thought of in terms of efficiency loss).

The situation that we have described is representative of situations where individuals do not have complete knowledge of their environment. Consequently, they are compelled, while choosing the future course of action, to use the limited information available to them. We capture this process of information diffusion (or information exchange) amongst individuals-a critical factor in technology adoption — by a (social) network. Individuals may start with a particular technology choice, but their future choice is governed partly by their own experience and partly by the experience of their acquaintances. In this case, we assume that individuals choose the technology that, according to their information, may lead to the most successful experience. The imitation behaviour of individuals is meant to encapsulate a rule-of-thumb that individuals use, possibly due to the complexity of the environment. We assume imitation of the most successful observable action (as opposed to other imitation dynamics such as imitation of the action that does best on average) because a successful action is more visible and prominent than, say, a successful-on-average action.

To answer the two questions posed above, we conduct a simulation study. For a given population size and average level of connectivity, we generate two network types that are relevant for social interactions: "scale-free" networks and "small-world" networks (described later in more detail). The reason behind choosing these two network types is that they resemble empirical data on networks-in "scale-free" networks, a few individuals are very well connected while most of the other individuals are not as well connected, as a result of which the average path length is low but connectivity is low at the same time; in small-world networks, the distance between two randomly chosen nodes grows proportionally to the logarithm of the number of nodes in the network. ${ }^{2}$ The initial assignment of actions $(P$ or $R$ ) amongst individuals is done randomly. Initial conditions may be important in shaping the final outcome due

2 Barabási and Albert [5] find that the way in which web pages in the internet are connected can be described by scale-free networks. Soramaki et al. [20] find that the network topology of the interbank payments transferred between commercial banks resemble scale-free networks-the network includes a tightly connected core of banks to which most other banks connect. Barabási and Bonabeau [6] present other examples of the occurrence of scale-free networks. On the other hand, Kogut and Walker [13] examine the network of cross-ownership amongst German firms and find evidence of it being described by small-world networks. Davis et al. [9] study the structure of the Fortune 1000 network of corporate directors and the company interlocks of a cohort of 195 Fortune 1000 firms from 1982 to 1999, and come to a similar conclusion. Fleming and Marx [10] studied inventors in Silicon Valley and Route 128 in Boston and find that the network of inventors has a small-world structure, while Newman [15] finds the same structure in co-authorship networks in biology, physics and mathematics. Uzzi et al. [22] discuss other empirically relevant occurrences of small-world networks. 
to path dependence, and in order to study their effect, the proportion of individuals who begin with action $P$ (or $R$ ) is varied. Then, the matching and imitation procedure described above is carried out until the first instance appears where all individuals choose the same actionhere, the society has reached a convention and adopted a particular action (or technology) as the standard.

As mentioned earlier, there are two features of this entire process that we intend to develop an understanding of: the relation between the network characteristics and the probability of reaching population-wide efficient coordination, and their influence on the time taken to reach coordination. We consider two categories of characteristics: factors that arise from network specific characteristics (NSC) and factors that arise due to the initial assignment of actions (IAA). Examples of NSC factors include the size of the network, the average degree or the average number of links per node (or individuals) in the network (this is indicative of the average connectivity in the network), the standard deviation in the number of links per node (this reflects the disparity in connectivity in the network) and the average centrality of the nodes in the network as given by the positional power. Examples of IAA factors include the share of nodes who are initially seeded with action $P$, their average number of links, their centrality and the extent to which they are segregated from others who have initially been assigned action $R$. We describe these factors in Sect. 2.

In this paper, we focus on the outcome of Stag-Hunt coordination games in the mediumrun. There is, on the other hand, a vast (theoretical) literature on the long-run outcome in such games due to imitation. ${ }^{3}$ Robson and Vega-Redondo [16] study coordination games with global random matching and global imitation and find that efficient coordination is achieved in the long-run for a large enough population. While global interaction is an element of our model, we stress on the "local nature" of individuals" decisions by emphasising the role of networks. In Alós-Ferrer and Weidenholzer [1], individuals located on a circle interact with the $k$ neighbours on either side and imitate the action that yields the highest payoffs. It is shown that the efficient equilibrium may be uniquely obtained. Noticeably, the interaction and information are both local in the sense of not encompassing the entire population. AlósFerrer and Weidenholzer [2] and Khan [12], on the other hand, disentangle information and interaction. The former analyses imitation behaviour in coordination games when individuals interact locally but obtain information from outside their neighbourhood, and find that large enough populations manage to coordinate efficiently. In contrast, the latter assumes that interaction is global, but information is locally obtained from the ties in the network, and shows that even though the efficient equilibrium is always stochastically stable, the inefficient equilibrium may co-exist in the long-run.

The papers referred to above add a noise component in the imitation procedure in order to identify the stochastically stable equilibrium. This equilibrium is relevant in the longrun and is independent of the IAA due to the noise component. In this paper, we adopt the same model as in Khan [12] but focus on the medium-run, which may be thought of as the time period where initial factors do not completely determine the outcome but still have a bearing on the process. This also makes for an interesting comparison of the determinants of the coordination process in the medium-run (in this paper) and the long-run (as in Khan [12]). The approach here is similar to Lee and Valentinyi [14], who analyse an error-free best-response dynamic for local interactions. We simulate and examine an error-free local imitation dynamic when the interaction is global.

There is also a vast literature that simulates coordination games on networks: individuals interact with their neighbours and their strategy choice is updated using some variant of the

3 Weidenholzer [25] reviews the theoretical literature on networks and coordination games. 
replicator dynamic or the best-response dynamic. Santos et al. [19] find that heterogeneity in the number of links makes efficient coordination more likely with a proportional imitation rule. Roca et al. [17] report that while the coordination outcome depends on the strategy updating rule and on the type of network, generally speaking, network clustering favours efficient coordination. However, for the case of myopic best-responses, Roca et al. [18] mention that the influence of the network is rather weak. This is in line with Buskens and Snijders [8], who also find that network structure does not play a major role for a range of the parameter space, but in the event that the network is important, the density of the network and the centralisation are important factors that promote efficient coordination. ${ }^{4}$ Similarly, Tomassini and Pestelacci [21] conclude that with a best-response dynamic, network structure may have a limited role, though in some network types, clustering may foster efficient coordination.

The important difference between the existing papers and our paper is that in the former, the network forms the basis for both interaction and strategy updating. This means that each node plays the coordination game with its neighbours and updates its action with a best-response or imitation rule that depends on the strategies or payoffs of the neighbouring nodes. We disentangle the interaction and informational roles of the network by assuming that interaction is global and random but imitation is local, in order to focus on the informational content of the network.

\section{Methodology}

The payoff matrix below describes the (Stag-Hunt) coordination game dealt with in this paper.

\begin{tabular}{l|cc} 
& \multicolumn{1}{c}{$P$} & \multicolumn{1}{c}{$R$} \\
\cline { 2 - 3 }$P$ & $a, a$ & $b, c$ \\
$R$ & $c, b$ & $e, e$ \\
\cline { 2 - 3 } & &
\end{tabular}

The conditions that we impose on the payoffs are: (1) $a>c, e>b$, (2) $a>e$ and (3) $c>b$. Condition (1) says there are two strict equilibria represented by the pairs $(P, P)$ and $(R, R)$; (2) says the equilibrium payoff of coordinating on $P$ dominates the payoff from the other equilibrium; and (3) says that in case of mis-coordination, it is preferable to choose $R .^{5}$

Individuals from a finite population are randomly matched to play this coordination game and receive payoffs depending on their own action and that of their randomly drawn co-player. After receiving payoffs, the individuals update their action for the next period by local imitation: each individual has a set of neighbours (this set includes the individual himself) whom he can observe. An individual imitates the action of the highest payoff receiving individual in his observation set. ${ }^{6}$ This continues recurrently until a convention is established. Our simulation study aims to identify characteristics that determine the likelihood of convergence

\footnotetext{
4 Antonioni et al. [3] conduct an experimental study of coordination games on networks with human subjects and find that there is no significant difference in the likelihood of efficient coordination in cliquish networks compared to random networks.

5 The literature on coordination games generally focuses on the tension between Pareto-dominant equilibrium and risk-dominant equilibrium. The payoffs assumed here deal with a wider class of coordination games: a risk-dominant coordination equilibrium must satisfy condition (3) above, but the converse may not be true.

6 Due to the imitate-the-best-neighbour dynamic, the precise magnitude of the payoffs is not relevant. Further, as all payoffs are disparate, there will be a unique action that an individual chooses.
} 
to the efficient equilibrium, and the time needed for this event to occur. Furthermore, we would like to identify the network characteristics that are the most prominent indicators of the emergence of efficient coordination.

In our simulations, we construct "scale-free" networks and small-world networks based on the methods developed by Barabási A and Albert [5] and Watts and Strogatz [23], respectively. For clarity, let us first describe the construction of a small-world network. Suppose we want to construct a network for $n$ nodes (where $n$ takes values 100, 200 and 300) with mean degree equal to $d$ (we choose $d$ to take the values 4,6 and 8). To begin with, all the nodes are arranged along the circumference of a circle and each node is connected to the nearest $\frac{d}{2}$ nodes on either side. Thereafter, each link is rewired node by node with an exogenously chosen probability, $p$, i.e. each of the links above is broken with this probability, and connected randomly to another node. The probability of relinking, $p$, takes all values from 0.00 to 1.00 with stepsize 0.10 . This procedure results in small-world networks, named so because the distance between two randomly chosen nodes in a network grows proportionally to the logarithm of the number of nodes in the network. We generate 100 different networks for each triplet $(n, d, p)$ to smoothen the randomness in this process.

The construction of "scale-free" networks is based on the method of preferential attachment, introduced by Barabási and Albert [5]. ${ }^{7}$ Suppose we want to construct a network for $n$ nodes and mean degree close to 4 . We begin with three nodes (say $i, j$ and $k$ ), each of which is connected to the other two. Next, we specify that each incoming node will be connected to exactly two other nodes, and the nodes to which this new entrant will be connected vary probabilistically with the degree of the existing nodes. So, the fourth node $(l)$ has equal probability of being connected to each of $i, j$ and $k$ (and $l$ is connected to exactly two of these three nodes), as $i, j$ and $k$ have the same number of connections (i.e. two). But now suppose that $l$ is connected to $i$ and $j$. Then the next entrant (node $m$ ) has a higher probability of being connected to $i$ and $j$ (as they have three links) as compared to $k$ and $l$ (who have two links). Entrants enter the network until we have the desired number of nodes in the network, with each entrant being more likely to be connected to a node with higher degree. In order to generate a network that has an average degree close to $d$, we begin with $\frac{d}{2}+1$ nodes that are all connected to each other, and then make each entrant add $\frac{d}{2}$ links to the network, such that the probability of linking to an existing node is in proportion to the degree of the existing nodes. This algorithm generates "scale-free" networks and average degree close to $d$.

Table 1 below describes our simulation strategy. We consider three population sizes, comprising of 100, 200 and 300 nodes. For each population size, we vary the average number of links per node to be 4, 6 or 8 for small-world networks, and approximately 4, 6 or 8 for "scale-free" networks. For small-world networks, as the network generation process is probabilistic due to stochastic rewiring, for each combination of network size, a particular number of average links per node and probability of rewiring, 100 networks are generated; consequently, 9900 networks are generated in total. On the other hand, for "scale-free" networks, as the network generation process is probabilistic due to preferential attachment, for each combination of network size and average number of links to be added per entrant, 100 networks are generated; consequently, 900 networks are generated in total. It is important

\footnotetext{
7 The networks we generate are scale-free on average - this is supported by the average degree distribution of the networks shown in Sect. 3.1. Even though our simulations seem to suggest that individual networks do capture the property that some nodes are highly connected while most are not, it is possible that an individual network does not exactly fit the scale-free property. We label these networks "scale-free" as we use the Barabási-Albert algorithm for generating them. At the same time, we note that this is only one method of generating scale-free networks and the network generated here may not be completely representative of the scale-free networks observed in the real-world.
} 
Table 1 Simulation parameters, number of random drawings and runs for small-world networks and "scalefree" networks

\begin{tabular}{lll}
\hline Parameter & $\begin{array}{l}\text { Small-world network } \\
\text { Values }\end{array}$ & $\begin{array}{l}\text { "Scale-free" network } \\
\text { Values }\end{array}$ \\
\hline Population size & 100,200 and 300 & 100,200 and 300 \\
Average number of links per node & 4,6 and 8 & 4,6 and 8 (approx.) \\
Relinking probability & $0.00,0.10,0.20$, & \\
& $0.30,0.40,0.50$, & \\
& $0.60,0.70,0.80$, & \\
Number of reconfigurations & 0.90 and 1.00 & 100 \\
Share of nodes seeded with $P$ & 100 & $0.02,0.05,0.10$, \\
& $0.02,0.05,0.10$, & $0.15,0.20,0.25$, \\
& $0.15,0.20,0.25$, & $0.30,0.35,0.40$ \\
Number of reconfigurations (per network) & $0.30,0.35,0.40$ & 100 \\
Number of initial configurations & and 0.45 & 900,000 \\
Number of runs per initial configuration & 100 & 100 \\
Total number of runs & $9,900,000$ & $90,000,000$ \\
\hline
\end{tabular}

to remark that the network specific characteristics (NSC, discussed later) are generated at this stage. Once the network is created, it is necessary to seed a fraction of the nodes with action $P$ (and the remaining with action $R$ ). The table indicates the proportion of nodes seeded with action $P$, for each of the 9900 (900) small-world ("scale-free") networks. While the proportion of initial $P$ nodes is fixed at 10 different values, the assignment is random, and we repeat the process of assignment 100 times for each of the proportions in the table. This leads to 9,900,000 $(900,000)$ potentially different initial small-world ("scale-free") network configurations. The characteristics that arise from the initial assignment of action (IAA, discussed below) are generated at this stage. Each configuration is run 100 times to account for the random matching assignment, leading to $990,000,000(90,000,000)$ simulations for small-world ("scale-free") networks.

After each configuration is created, we simulate the process of playing the coordination game under global random interaction and local imitation in the following way. Each node is randomly matched to another node. The action profile of the matched nodes yields the payoffs in accordance to the payoff matrix presented earlier. After the matching and payoff realisation process is completed, the action updating phase begins. Each node looks at the payoffs and actions of its neighbours based on the network configuration (we assume nodes to be a part of their own neighbourhood) and adopts the action that yielded the highest payoff in its respective neighbourhood. After each node has updated its action in this manner, the process of random matching continues until we reach a state where all nodes have the same action (i.e. a convention has been established) — clearly, there can be no further change in the action chosen by a node beyond this point.

We collect data on the particular convention established $(P$ or $R)$, and the number of times we have to conduct the random matching until the first instance that all nodes choose the same action (the convergence time). As can be seen from Table 1, to account for the randomness in the matching process, for a given network and given IAA, each initial configuration is played out 100 times. The data on the convention established and the convergence time are 
aggregated at this level, i.e. the data collected indicate the fraction of times in the 100 runs of the random matching process that efficient coordination is achieved for a given network and given initial conditions, while the mean convergence time is the average number of iterations of random matching needed to converge to a convention for a given network and given initial action assignments.

Before discussing the results, we describe the factors included in the analysis. As mentioned earlier, they can be categorised into factors that arise from the network (NSC) and factors that arise from IAA.

First, the NSC factors. These are:

(i) Size The number of nodes. Since we have only three different network sizes, we treat this as a categorical variable and interpret the networks with 100, 200 and 300 nodes as small, medium and large networks. The medium-sized network, with the number of nodes equal to 200, is the baseline, and we examine the effect of a smaller (larger) population on efficient coordination.

(ii) Degree (mean, quintiles and standard deviation) This is the average number of links of a node in the network, the quintiles of the degree distribution and the standard deviation in the degree of the nodes. For small-world networks, by construction of the network, the average degree takes only three values (i.e. 4, 6 and 8). Networks where each node has degree 4, 6 and 8 are interpreted as networks with low, medium and high connectivity. On the other hand, for "scale-free" networks, the average degree of the network where each entrant adds 2 links and the number of nodes is equal to 100, 200 and 300 is 3.94, 3.97 and 3.98, respectively. We group these networks types together and interpret them as networks with low connectivity. Similarly, the average degree of the network where each entrant adds 3 (4) links and the number of nodes is equal to 100, 200 or 300 is 5.88, 5.94 or 5.96 (7.80, 7.90 or 7.93$)$, respectively; we label these networks as networks with medium (high) connectivity. The network with medium connectivity is the baseline, and we examine the effect of a network with greater (lesser) connectivity on efficient coordination. The standard deviation and quintiles of the degree distribution are treated as a continuous variable.

(iii) Probability of relinking (for small-world networks) The small-world networks are generated by a process of probabilistic rewiring of existing links of the network where the nodes are arranges on a circle and connected to $k$ neighbouring nodes on either side. As the probability of rewiring increases, there is greater randomness in the structure of the network.

(iv) Positional power (mean, quintiles and standard deviation) In general, the influence of a node on the entire population depends not only on its own connectivity but also on the connectivity of the nodes it is connected to. The degree variable above only looks at the number of direct connections of a node while ignoring the strength of indirect connections when in fact being connected to a well-connected node may be substantially different from being connected to a node that is not well connected. The variable "positional power" (or power) takes into account the global structure of the network. To incorporate this feature, we use the long-path method, which is a global positional measure of a node (from Wei [24] and Kendall [11]). ${ }^{8}$ After constructing

8 More formally, if there are $n$ nodes, $A$ represents the $n \times n$ neighbourhood or adjacency matrix, $x^{0}$ is the $n$-vector of ones and $x^{t}=A x^{t-1}$, then the long-path vector is given by $\lim _{t \rightarrow \infty} \frac{x^{t}}{\sum_{i} x_{i}^{t}}$. This measure is equivalent to the principal eigenvector of the neighbourhood matrix $A$, i.e. it is a solution $\lambda$ of the equation $\lambda x=A x$. 
this power measure, we look at mean, quintiles and standard deviation of the positional power across nodes.

Next, we present the IAA factors. These are:

(i) Share of $P$ nodes This is the fraction of nodes that are assigned action $P$ while setting up the initial conditions.

(ii) Degree of $P$ nodes (mean, quintiles and standard deviation) This refers to the average degree and the standard deviation in degree of the nodes who have been given action $P$ in the initial assignment.

(iii) Positional power of $P$ nodes (sum, mean, quintiles and standard deviation): As in (iv) of NSC factors above, we want to look into the impact of nodes being connected to well-connected nodes. From the power statistic, we derive the sum of the positional power of the seed $P$ nodes, their mean power and the standard deviation in power across such nodes.

(iv) Segregation of $P$ nodes (normalised) This statistic is used to examine the extent to which the seed $P$ nodes are segregated in the population using a measure developed by [4]. They define the segregation measure as the probability that a node of a certain type (in this case of the type holding action $P$ ), after a random walk along the links of the network, where the walk terminate at every step with an exogenously given probability, terminates this walk at a node of the same type. The exogenously probability of termination that we use is 0.15 , which implies that the expected number of steps in a random walk is approximately 6 . As this measure is not invariant to the size of the network, [4] develop a normalised measure that is invariant in this respect. ${ }^{9}$

(v) Segregation of $R$ nodes (normalised) We construct a similar measure for seed $R$ nodes.

It is important to note that after the initial conditions are assigned and the process of random matching and local imitation is started, the measures indicated by IAA (i)-(v) will change as the nodes update actions by local imitation. However, since we are interested in understanding the effect of initial conditions, we only use the measures as generated from the initial network and its initial distribution.

The variables that we want to explain by the above-mentioned factors are:

1. Payoff dominant wins The proportion of times that the population coordinated on the payoff-dominant (or efficient) equilibrium for a given network and given set of initial conditions (i.e. holding fixed the network that is generated for a given population size, degree per node, relinking probability and holding fixed the IAA).

2. Conditional mean convergence time We continue the random matching and local imitation process until the population coordinates on an equilibrium, and the convergence time is given by the number of iterations of the random matching process until convergence. The mean convergence time indicates the average time for coordination (to any equilibrium)

\footnotetext{
9 More formally, let $A$ be the $n \times n$ neighbourhood matrix defined on the set of $n$ nodes by the links of the network (i.e. $a_{i j}=1$ if there is a link between the two nodes $i$ and $j$, and $a_{i j}=0$ otherwise) and $L$ be the $n \times n$ row-stochastic matrix associated with matrix $A$ such that $l_{i j}=\frac{a_{i j}}{\sum_{k} a_{i k}}$. If $\alpha$ is the continuation probability of the random walk, and the $n \times n$ matrix $Q$ is such that $q_{i j}$ represents the probability that this stochastic random walk ends in node $j$ when it started in node $i$, then $Q=(1-\alpha)(I-\alpha L)^{-1} L$. The segregation measure for $P$ nodes is given by $d_{P}^{T} Q c_{P}$, where $c_{P}$ is a $1 \times n$ column vector such that $c_{1 i}=1$ if the $i$ th node takes action $P$ and 0 otherwise, and $d_{P}$ is a $1 \times n$ column vector such that $d_{1 i}$ is the inverse of the number of $P$ nodes if the $i$ th node takes action $P$ and 0 otherwise. As this measure is not invariant to population size, the normalised segregation index for $P$ nodes is defined as $s^{-1} d_{P}^{T} Q c_{P}$, where $s^{-1}$ is the inverse of the share of $P$ nodes in the entire population.
} 
for a given network and given its initial conditions (i.e. holding fixed the network that is generated for a given population size, and holding fixed the IAA). Recall that for any given network and any given set of initial conditions, the process of random matching and local imitation goes over 100 runs, and the average time is taken over these 100 runs. Since our interest lies in the time taken to attain efficient coordination, we only examine the mean average time, conditional on the observed proportion of efficient coordination being at least 0.75 .

The choice of the NSC and IAA factors included in the analysis is mainly guided by two considerations. Firstly, the parameters of the simulation such as the size of the population, the average degree, the probability of relinking (for small-world networks) and the initial share of $P$ users are all included. Secondly, we have included network properties that are most relevant from the perspective of an imitation dynamic. For an imitation dynamic, observability is arguable the most important driver-how many individuals can one observe and how many individuals observe a given individual, how easy or difficult is it for an individual to observe individuals using a different action (as only then may one consider switching actions). These considerations have motivated us to focus on the local connectedness-related properties of the network (such as average degree), the global connectedness properties (such as positional power) or ease of observing nodes using the other action (such as segregation index). ${ }^{10}$

We use a linear regression model to identify the factors that influence the local imitation process. After having done so, we attempt to provide a first answer to the question of the relative importance of these factors. In other words, if it were possible to create a hierarchy of factors by their influence on the local imitation process, which factor would be at the top of the pyramid? For this purpose, we use a classification tree algorithm (described later) to see whether it is possible to predict the outcome of the simulations based on the NSC and IAA measures. Finally, we investigate whether efficient coordination is more probable, and whether it is faster, in a particular type of network (i.e. "scale-free" or small-world). In order to do so, we look at the empirical distributions of the proportion of times efficient coordination is achieved, and for the time taken to achieve efficient coordination, and test whether they are statistically different, following which we use a linear regression model to check for the effect of network type on these two variables of interest.

Before discussing the results, we address two relevant aspects of the imitation dynamic. Firstly, since we impose an imitate-the-best-neighbour dynamic on the individuals, one observation of an individual receiving payoff $a$ (in reference to the payoff matrix) is sufficient to induce imitation of action $P$ in the neighbourhood. This is the only event when action $P$ will be imitated in the neighbourhood. On the other hand, imitation of action $R$ will occur either when it is the only action in the neighbourhood or in case of neighbourhoods that have both actions, the action $P$ individuals are matched to action $R$ individuals. Thus, for imitation of action $R$ in a mixed neighbourhood, the random matching process needs to be unfavourable for all action $P$ individuals, whereas for imitation of action $P$, the matching process needs to be favourable for only one such action $P$ individual. Secondly, we exclude the possibility of an individual inferring the action of his randomly chosen co-player from his own payoff, thereby opening up the possibility of an individual imitating co-players even if they are not neighbours. We purposely exclude this possibility because our focus is on how the network

\footnotetext{
10 Factors such as the clustering coefficient have also been observed to be important in the existing literature. While these features have not been explicitly included due to the computational complexity involved in obtaining the value of these variables, some of the included variables capture a part of the effect of these excluded factors. For example, the probability of relinking captures a part of the clustering effect-as the probability of relinking increases, it is expected that the clustering coefficient will decrease.
} 
structure affects the coordination process, and imitation of a co-player who is not a neighbour is an extraneous force from the perspective of the objective of this study.

\section{Results}

In Sect. 3.1, we present an overview of the degree properties of the "scale-free" and smallworld networks that were generated. Sections 3.2 and 3.3 discuss the results pertaining to the frequency of attaining efficient coordination and the time taken to attain efficient coordination, respectively. In Sect. 3.4, we compare the frequency of attaining efficient coordination and time taken for doing so in small-world and "scale-free" networks.

\subsection{Properties of Generated Networks}

Table 2 shows the on-average degree distribution of the "scale-free" networks and the smallworld networks generated. For ease of presentation, we present only the smallest and largest network sizes (i.e. with 100 and 300 nodes), and the smallest and largest average degree (equal to approximately 6 and 8). In case of "scale-free" networks, for each population size and average degree, 100 networks are generated. Similarly, in case of small-world networks, for each population size, average degree and probability of rewiring, 100 networks are generated. Thus, in the latter case, for each population size and average degree 1100 networks are generated. We present the average degree distribution over these 100 networks for "scalefree" networks and 1100 networks for small-world networks for network size equal to 100 and 300 , and average degree equal to 4 and 8 .

Table 2 allows for a comparison of the degree distribution across the two different network classes given network size and average degree, and across different network sizes/average degree holding the network class and average degree/network size constant. For a given network size and average degree, the general trend is that the degree distribution up to the 75 th percentile is on average lower for "scale-free" networks than for small-world networks. However, the maximum degree is on average substantially higher for "scale-free" networks. Thus in the "scale-free" networks generated, most of the nodes are not as well linked (at least in comparison to small-world networks), but there exist few nodes which are highly connected.

Within "scale-free" networks, for a given population size, the standard deviation is higher for larger average degree (i.e. the dispersion is larger); on the other hand, for a given average degree, an increase in the population size increases the dispersion primarily at the higher end of the degree distribution - the average maximum degree is substantially higher for a larger population size, whereas the lower quartiles are largely similar. Within small-world networks, for a given population size, the standard deviation of the degree distribution does not seem to be too extreme as the average degree changes; a similar comment applies when one varies the population size while holding the average degree constant. ${ }^{11}$

\subsection{Efficient Coordination}

The variable Payoff dominant wins captures the proportion of times that convergence to the efficient action is achieved for a given network size, average degree, relinking probability for small-world networks, and a given distribution of initial actions. The process of random

\footnotetext{
11 We also find, as expected, that for small-world networks, the standard deviation in degree varies positively with the probability of relinking.
} 


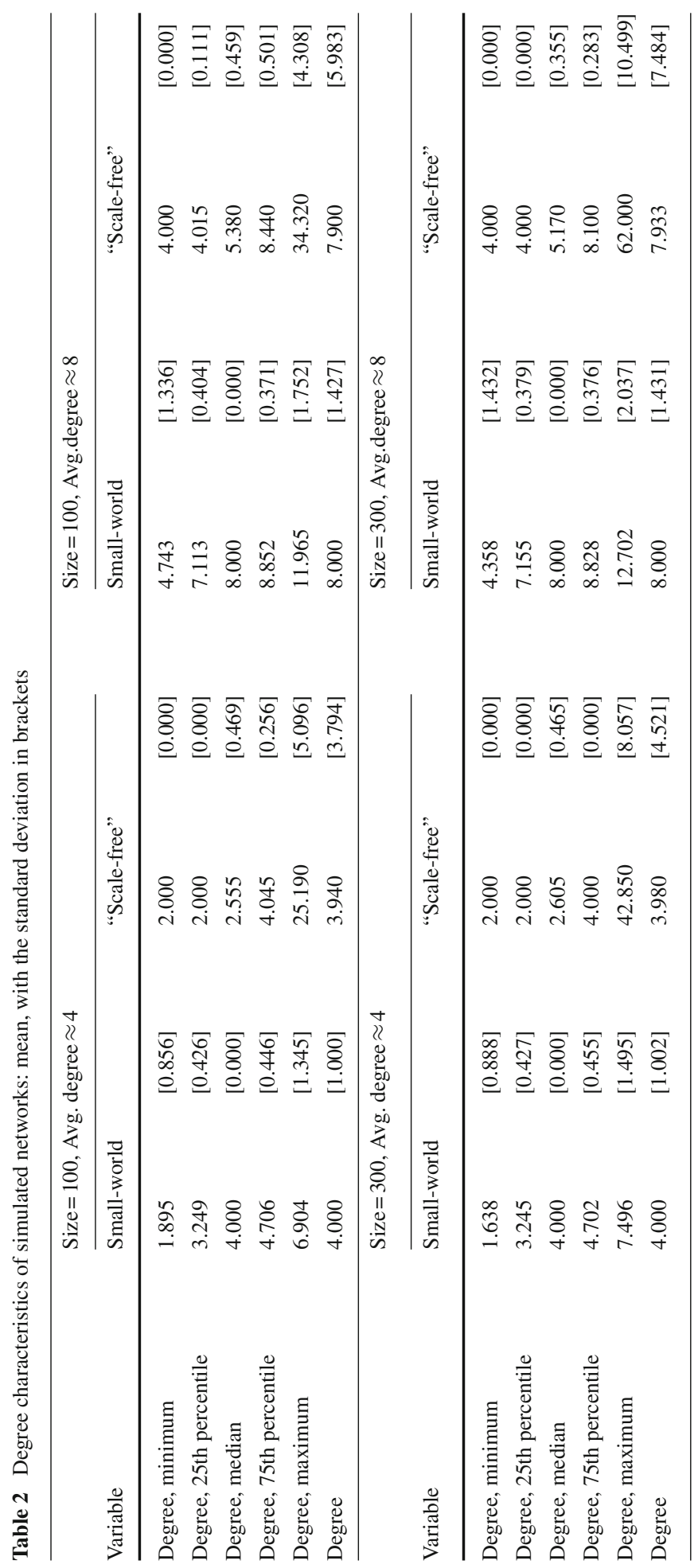



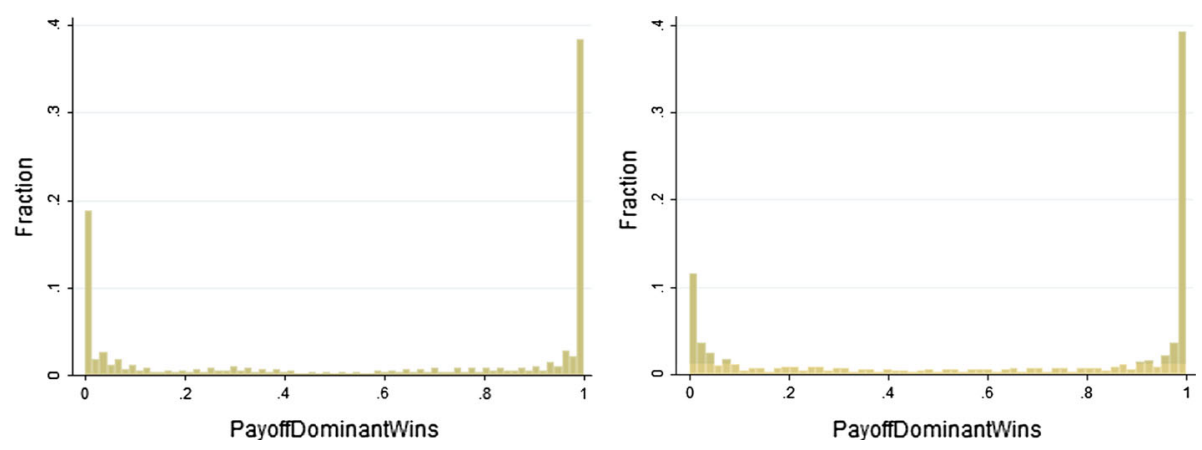

Fig. 1 Distribution of proportion of times efficient coordination is achieved: small-world (left) and "scalefree" (right)

matching till convergence is repeated 100 times, and the variable Payoff dominant wins is the proportion of times efficient coordination is achieved in these 100 repetitions. The total number of observations is 9,899,069 $(900,000)$ for small-world ("scale-free") networks (see Table 1). ${ }^{12}$

The mean value of Payoff dominant wins is 0.6000 for the small-world networks and 0.6373 for the "scale-free" networks, with corresponding standard deviations of 0.4285 and 0.4071, respectively. Figure 1 shows the distribution of the values for Payoff dominant wins for both types of networks.

The figure reveals that the two most frequent outcomes are convergence to efficient coordination with proportion close to zero or one. These extreme outcomes are almost exclusively driven by the share of seed $P$ nodes: For low (high) values of the share of $P$ nodes, the process does not exhibit sufficient variability and converges almost always to the inefficient (efficient) equilibrium. Figure 2 shows the distributions that result after restricting the data to share values of $0.10,0.15$ and 0.20 for both small-world networks and "scale-free" networks. We see that more variability is obtained when restricting to these intermediate values of the share of $P$ nodes.

This leads to a first observation that the initial share of action $P$ or action $R$ adopters heavily influences the direction taken by the coordination process. When the share of the initial adopters of action $P$ is sufficiently high (low), one expects other (network) features to not be very germane to the outcome of the process. As a result, for further analysis, we focus on a subset of the data where the initial share of action $P$ adopters is such that the outcome of the coordination process exhibits some amount of variability; specifically, we consider the case where the share of initial action $P$ adopters equals $0.15 .^{13}$

12 The total number of observations generated for small-world networks equals $9,900,000$, but we use $9,899,069$ for the following analysis. This is because the process of random interaction and local imitation is aborted if convergence to either equilibrium does not happen within 100 iterations, and there are 931 such instances. Interestingly, such instances of non-convergence to an equilibrium occur most frequently for middling values of the initial share of $P$ nodes - more than two-thirds of such cases occur when the share is equal to 0.2 or 0.25 . This is most probably because if the share is "too high (low)" then the process would converge to the (in)efficient equilibrium within 100 time periods. Further, understandably, the non-convergence occurs predominantly (i.e. more than two-thirds of the time) for lowest average degree (equal to 4) or largest population size (equal to 300); almost half of the times this happens for the lowest average degree and largest population size. However, this forms only approximately $0.001 \%$ of the data set, and may be ignored without severe consequences for the analysis.

13 A second reason is that isolating a single value of share permits a cleaner examination of other factors that influence the process. 

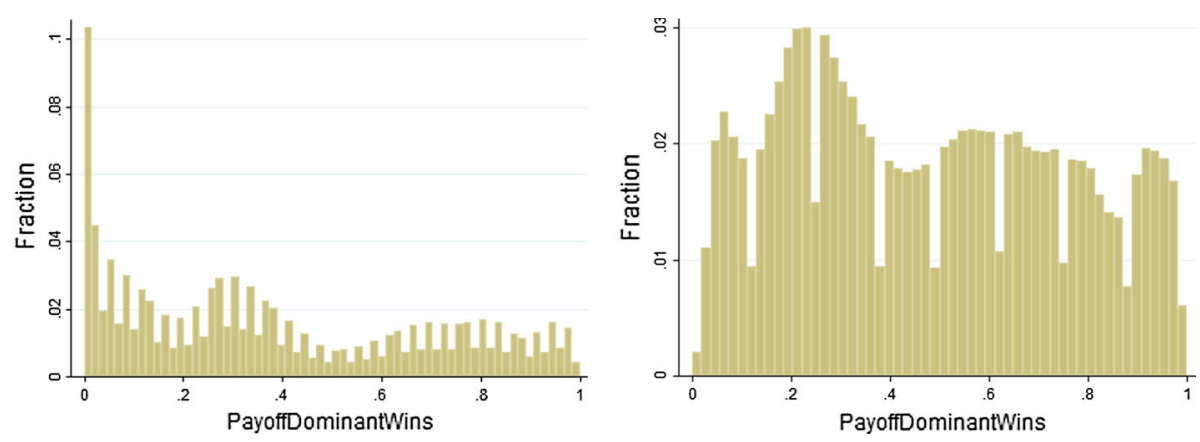

Fig. 2 Distribution of proportion of times efficient coordination is achieved when share of $P$ nodes equal to 0.10, 0.15 and 0.20, small-world networks (left) and "scale-free" networks (right)

To analyse the effect of NSC and IAA factors on the likelihood of efficient coordination, we run an ordinary least squares regression on the dependent variable (Payoff dominant wins) with the other factors mentioned in the previous section as the explanatory variables. In the analysis, apart from holding the share of initial action $P$ adopters fixed, we first hold the size of the network and the average degree constant (at values of 200 and 6, respectively) and thereafter examine the effect of a larger (smaller) network and higher (lower) average connectivity on the convergence process. This allows us to first control for three inputs of the process (size, average degree and initial share of adopters) and then examine other factors that influence this process. The result of the analysis is displayed in Table $3 .{ }^{14}$

We discuss the factors that have a significant (at the 0.001 level) effect on the possibility of efficient coordination.

(i) Probability of rewiring (for small-world networks): A possible reason for the positive effect of this variable on efficient coordination is that the increase in the probability of rewiring increases the variance in degree across nodes. Due to rewiring, some of these nodes are better connected and form "hubs", while other nodes are not as well connected. These hubs are relatively more resistant to $R$ for the reason that a greater number of nodes will now have to be affected simultaneously by an unfavourable matching for the hubs to imitate $R$. On the other hand, the hubs can very rapidly imitate to $P$ by imitation as they observe a higher number of nodes and they need only one successful $P$ individual to imitate. Further, once these hubs imitate $P$, their influence extends over a larger number of nodes, who may then also imitate $P$.

(ii) Degree distribution of nodes (for "scale-free" networks): The on-average maximum degree of the network has a negative effect on efficient coordination. A possible reason for this might be that "scale-free" networks are endowed with some amount of asymmetricity and that any further increase in asymmetricity (in the degree of nodes) may impede efficient coordination due to the development of isolated pockets from where the inefficient action may spread.

(iii) Distribution of power of nodes (for small-world networks): The median and upper quintile of positional power of the nodes exerts a positive effect on efficient coordination

\footnotetext{
14 In order to avoid the problem of multi-collinearity, the correlation between the independent variables was checked. In case the correlation was greater than 0.65 , we eliminated one of the independent variables by the following procedure: if one of the two variables is a parameter (such the relinking probability in case of small-world networks), it is retained and the other variable is dropped; in case none of the variables are parameters, the variable that correlates stronger with the dependent variable is retained. Robust standard errors are used to circumvent the issue of heteroscedasticity.
} 
Table 3 Factors that influence the likelihood for the process to converge to the efficient equilibrium state in small-world networks and "scale-free" networks of size 200, average degree equal to 6 (approximately) and initial share of action $P$ adopters equal to 0.15

\begin{tabular}{|c|c|c|c|c|}
\hline \multirow[b]{2}{*}{ Variable } & \multicolumn{2}{|c|}{ Small-world networks } & \multicolumn{2}{|c|}{ "Scale-free" networks } \\
\hline & Coef. & $\begin{array}{l}\text { Robust } \\
\text { SE }\end{array}$ & Coef. & $\begin{array}{l}\text { Robust } \\
\text { SE }\end{array}$ \\
\hline Probability of relinking & $0.0797^{*}$ & $(0.0008)$ & & \\
\hline Degree, maximum & & & $-0.0004^{*}$ & $(0.0001)$ \\
\hline Power of nodes, median & $8.9099^{*}$ & $(1.4421)$ & & \\
\hline Power of nodes, 75 th percentile & $43.1495^{*}$ & $(0.8331)$ & & \\
\hline Power of nodes, SD & & & $-25.7609^{*}$ & $(4.2795)$ \\
\hline Degree of $P$ nodes, 25 th percentile & $-0.0136^{*}$ & $(0.0005)$ & & \\
\hline Degree of $P$ nodes, median & & & $0.0048^{*}$ & $(0.0012)$ \\
\hline Degree of $P$ nodes, 75 th percentile & $0.0101^{*}$ & $(0.0004)$ & & \\
\hline Degree of $P$ nodes, mean & & & $0.0454^{*}$ & $(0.0007)$ \\
\hline Power of $P$ nodes, median & $9.3713^{*}$ & $(0.6487)$ & $5.8318^{*}$ & (1.1986) \\
\hline Power of $P$ nodes, 75 th percentile & $1.7767^{*}$ & $(0.4708)$ & $5.7737^{*}$ & $(0.8381)$ \\
\hline Power of $P$ nodes, maximum & $2.7374^{*}$ & $(0.1240)$ & & \\
\hline Segregation (norm.) of $P$ nodes & $-0.0528^{*}$ & $(0.0020)$ & & \\
\hline Segregation (norm.) of $R$ nodes & $-2.6095^{*}$ & $(0.0348)$ & & \\
\hline Constant & $2.6650^{*}$ & $(0.0392)$ & $.3155^{*}$ & $(0.0189)$ \\
\hline Number of obs. & 109,985 & & 10,000 & \\
\hline R-squared & 0.5775 & & 0.4771 & \\
\hline
\end{tabular}

The stars indicate significance at $0.1 \%$ level

in small-world networks, implying that nodes with better connections (taking into account both immediate and indirect connections) facilitate the contagion of action $P$.

(iv) Standard deviation of power of nodes (for "scale-free" networks): This has a negative effect on efficient coordination, due to reasons similar to (ii) above.

(v) Degree distribution of initial action $P$ nodes (for small-world networks): The sign of the coefficients of "Degree of $P$ nodes, 25th percentile" and "Degree of $P$ nodes, 75th percentile" suggest that efficient coordination is enabled by higher connectivity on the right tail and lower connectivity on the left tail of the degree distribution of the initial action $P$ nodes. This may be because such a realignment of links from the left tail of the distribution to the right tail enables formation of "hubs", which for reasons similar to (i), increases the chance on efficient coordination.

(vi) Degree distribution of initial action $P$ nodes (for "scale-free" networks): An increase in the links of the action $P$ node with median degree has a positive effect on coordination, on account of increased visibility.

(vii) Mean degree of initial action $P$ nodes (for "scale-free" networks): This has a positive effect on efficient coordination, due to higher average connectivity of the initial $P$ nodes.

(viii) Distribution of (positional) power of initial action $P$ nodes: The positive effect on efficient coordination is due to greater global connectivity (and thus global reach) of the action $P$ nodes. 
(ix) Normalised segregation index of $P$ nodes (for small-world networks): This has a negative effect on convergence to the efficient coordination outcome. If this segregation index is high, then it implies that a $P$ node has a high probability of meeting another randomly chosen $P$ node on a random walk along the links of the network. This implies that neither the $P$ node (which starts on the random walk) nor its neighbours share many links with $R$ nodes. This signifies that initial action $P$ users are not very "visible" to action $R$ users, reducing the possibility of efficient coordination.

(x) Normalised segregation index of $R$ nodes (for small-world networks): The reason for the negative effect of this variable on efficient coordination is similar to the one stated in (ix) above. A high segregation of $R$ nodes implies that $R$ nodes are, in general, not likely to observe (many) $P$ nodes, which locks them into the use of action $R$.

Thus far, the analysis has been conducted while fixing the size of the network, the average degree of the nodes in the network and the share of the initial action $P$ adopters. Here, we discuss the effect of these three parameters. We have already mentioned that the share of initial adopters is one of the most crucial determinants to the extent that if this share is below or above a threshold, then other factors tend to be not so relevant to the outcome of the process.

We assess the effect of size by comparing the baseline situation (where size equals 200, average degree equals 6 and share of initial action $P$ adopters equals 0.15 ) to two alternative situations where only the size of the network changes to 100 and 300 , respectively. We find a positive effect for size, which may be because for the same initial share of adopters and average degree distribution, a higher population size increases the probability of two action $P$ nodes being matched - an event which is necessary and sufficient for the spread of action $P$.

Similarly, a comparison of the baseline situation to two alternative situations where only the average node degree changes to 4 and 8 reveals that an increase in the average node degree increases the possibility of efficient coordination by increasing the on-average observation span of the nodes - an event which can only enable efficient coordination by bringing successful instances of the use of action $P$ within vision. ${ }^{15}$

In order to determine the factor that is "most" predictive or decisive concerning the likelihood for a society to be able to converge to the efficient state, we ran a classification tree algorithm on the data set of our network simulations. A classification tree analysis is a data mining technique to create a model that predicts the value of a target value (in our case the likelihood to converge to the efficient state) based on several input variables (in our case the NSC and IAA factors). The algorithm returns a binary tree that partitions the data on basis of the input variables (by a recurrent splitting of the data on basis of the values of the input variables) such that the parts are most conclusive in explaining the value of the target value. The hierarchy of the generated tree provides information on the order of importance of the input variables towards a conclusive partitioning. ${ }^{16}$

For our classification tree analysis, we used the in-built Matlab algorithm classregtree with the likelihood to converge to the efficient state as the output variable and all NSC and

\footnotetext{
15 The qualitative results displayed are relatively robust to a change in size of the network or change in average degree. The exception for "scale-free" networks is that Power of $P$ nodes, 75th percentile, ceases to be significant when all sizes are aggregated and that the maximum degree of nodes has a positive effect when the average degree decreases to $4-$ in this case, the median node of $P$ nodes has a negative effect. The exceptions for small-world networks are that the 75th percentile for action $P$ nodes ceases to be significant when the average degree increases, and median power of nodes has a negative effect for lower average degree (equal to 4).

16 For more information on classification tree analysis, see Breiman et al. [7].
} 


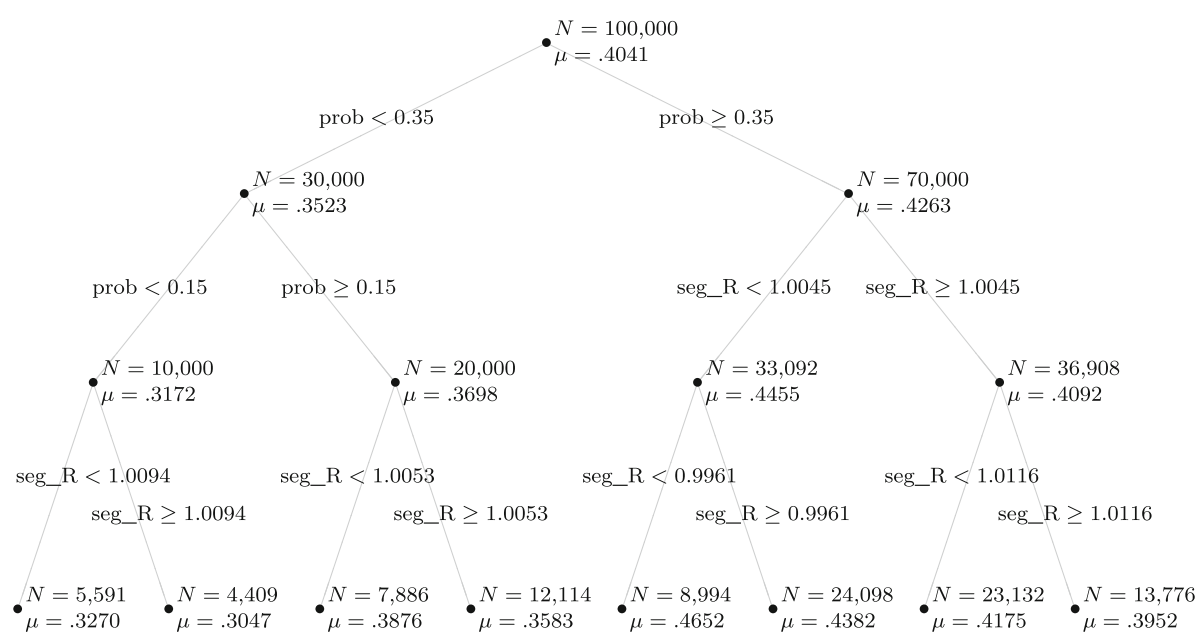

Fig. 3 Classification tree for the small-world network

IAA factors listed in Sect. 2 as input variables. Figures 3 and 4 present a pruned version of the outcome for, respectively, the small-world and the "scale-free" networks. For these figures, the data have been restricted to a network size of 200, a mean degree of 6 and share of initial action $P$ seeded nodes equal to 0.15 , and for the small-world network to those with strictly positive relinking probability. The main findings reported below are robust towards changes in either of these values and are also found when taking random subsamples of the data.

For the small-world networks (Fig. 3), we see that the most important variable is the relinking probability. This variable appears in the first layer and in one of the two branches at the second layer. When repeating the analysis while restricting the data to different parameter values of size, degree and initial share of $P$ nodes, this variable is in some occasions replaced by the standard deviation of the degree distribution, which is a variable that correlates highly with the relinking probability (correlation coefficient $\rho=0.9182$ ). The second variable that appears prominently is the segregation of the $R$ nodes. Where in the original dataset the overall likelihood to converge to the efficient state equals $40.41 \%$, after three splits the smallest and largest likelihood to converge to the efficient state over the eight partitions equals 30.47 and $46.52 \%$. We see that when data are split on basis of the segregation of the $R$ nodes, the part of the data with the lower segregation score has a larger likelihood to converge to the efficient state. This is consistent with the negative sign in the regression (see Table 3).

For the "scale-free" networks (Fig. 4), the two most important variables are the mean degree of the $P$ nodes and the segregation of the $R$ nodes. The importance of these two variables does not change when repeating the analysis while restricting the data to different parameter values of size, degree and initial share of $P$ nodes, but their internal ranking on importance is swapped for some specifications (in particular, for higher and lower initial share of $P$ nodes). From the numbers at the nodes, we see that a higher local connectivity of the $P$ nodes leads to an increase in the likelihood to converge to the efficient outcome-again this effect is consistent with the results of the regression that are reported in Table 3 . The effect of a split on basis of the segregation of the $R$ nodes is similar to that for small-world networks. 


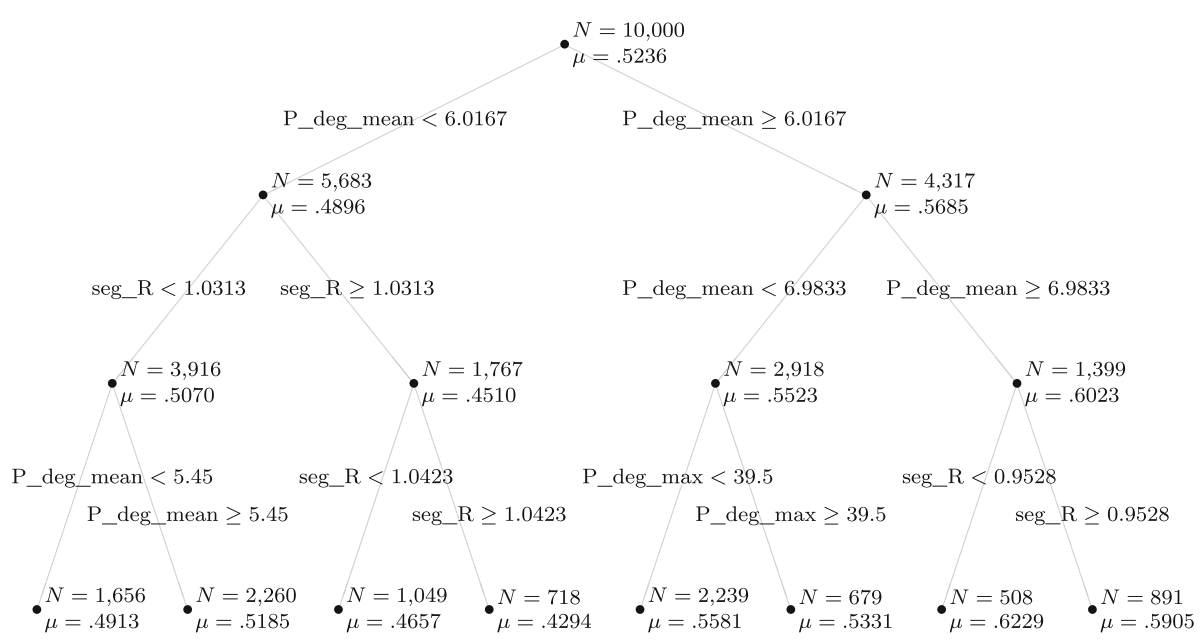

Fig. 4 Classification tree for the "scale-free" network
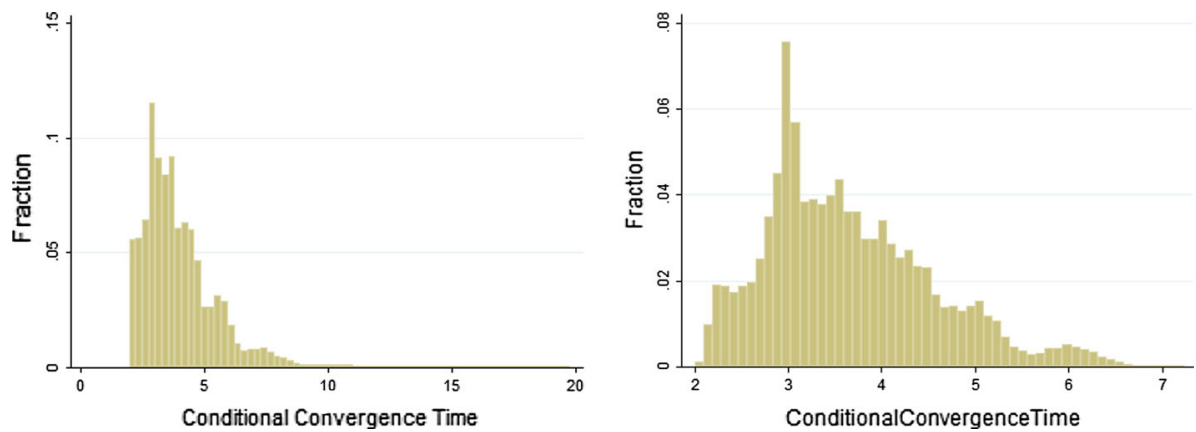

Fig. 5 Distribution for time taken to achieve efficient coordination (averaged over 100 runs): small-world (left) and "scale-free" (right)

\subsection{Time Taken to Achieve Efficient Coordination}

In this subsection, we analyse the time taken to coordinate on the efficient equilibrium. The importance of this issue stems from the fact that an earlier coordination on the efficient equilibrium avoids mis-coordination costs. The variable that records the mean convergence time (aggregated at the level of the 100 iterations of random matching) does so for both efficient and inefficient coordination. Since our interest lies in the time taken to attain efficient coordination, we exclude from the regression set the observations for which efficient coordination was attained less than three-fourth of the times. Thus, we only use the data where Payoff dominant wins is at least 0.75 . This leaves us with 5,265,575 (53.19\%) observations for small-world networks, and 501,081 (55.61\%) observations for "scale-free" networks. Across these observations, the mean number of iterations needed for the system to converge to one of the equilibrium states equals 4.0706 for small-world networks and 3.6590 for "scalefree" networks, with corresponding standard deviations of 0.9100 and 3.6590, respectively. The histograms in Fig. 5 display the distributions of the time taken to achieve coordination, conditional on the process to converge to the efficient outcome being at least 0.75 . 
Table 4 Factors that influence the time needed to reach efficient coordination for small-world networks and "scale-free" networks (conditional on the efficient equilibrium being reached at least three-fourth fraction of the time) for share equal to 0.20 , size equal to 200 and average degree equal to 6

\begin{tabular}{|c|c|c|c|c|}
\hline \multirow[b]{2}{*}{ Variable } & \multicolumn{2}{|c|}{ Small-world } & \multicolumn{2}{|c|}{ "Scale-free" } \\
\hline & Coef. & $\begin{array}{l}\text { Robust } \\
\text { SE }\end{array}$ & Coef. & $\begin{array}{l}\text { Robust } \\
\text { SE }\end{array}$ \\
\hline Probability of relinking & $-0.4376^{*}$ & $(0.0184)$ & & \\
\hline Degree of nodes, minimum & $0.2594^{*}$ & $(0.0090)$ & & \\
\hline Degree of nodes, maximum & $-0.1382 *$ & $(0.0040)$ & $-0.0064^{*}$ & $(0.0004)$ \\
\hline Power of nodes, maximum & & & $8.5478^{*}$ & $(0.6222)$ \\
\hline Degree of $P$ nodes, stdev & & & $-0.0641^{*}$ & $(0.0008)$ \\
\hline Segregation (norm.) of $P$ nodes & $1.7332^{*}$ & $(0.0643)$ & & \\
\hline Segregation (norm.) of $R$ nodes & $13.7371^{*}$ & $(0.2948)$ & & \\
\hline Constant & $-9.1758^{*}$ & $(0.3617)$ & $5.0500^{*}$ & $(0.0148)$ \\
\hline Number of obs. & 77,061 & & 9473 & \\
\hline R-squared & 0.4633 & & 0.4377 & \\
\hline
\end{tabular}

The stars indicate significance at $0.1 \%$ level

We use a linear regression model to estimate the average time taken for efficient coordination, the results of which are presented in Table 4. In this analysis, we fix the share of initial adopters at 0.20 , the size at 200 nodes and the average node degree at 6 (approximately). We discuss the factors that have a significant (at the 0.001 level) influence on the time taken for such coordination.

(i) Probability of rewiring (for small-world networks): An increase in the probability of rewiring reduces the conditional convergence time. A possible reason for this effect may be that an increase in the probability of rewiring is expected to lead to higher variation in nodes degree (thereby implying that some nodes have greater connectivity at the expense of others), and when the standard deviation of nodes increase, some of these nodes are better connected and form "hubs", while other nodes are not as well connected. For reasons alluded to earlier, these hubs are relatively more difficult to change to action $R$, and at the same time, more susceptible to action $P$. Additionally, they can more easily induce other nodes to imitate action $P$.

(ii) Degree of nodes, minimum (for small-world networks): An increase in the average number of links of the nodes with least connectivity increases the conditional convergence time. A reason might be that the increase in the average number of links of the nodes with least connectivity is at the cost of the links of the nodes with higher connectivity (as everything else is held constant). If nodes with higher connectivity are important for efficient coordination and the speed at which it occurs, such a realignment may lead to a longer convergence time.

(iii) Degree of nodes, maximum: An increase in the connectivity of nodes in the right tail of the degree distribution of the network reduces convergence time, for possibly the same reason outlined in (i) above, i.e. for efficient coordination, nodes with high connectivity are more crucial than an even spread of connectivity across nodes.

(iv) Power of nodes, maximum (for "scale-free" networks): This factor has a positive effect on the process of efficient coordination. When nodes have more positional power, they are in a better position to directly or indirectly access highly connected nodes, and this 
expedites efficient coordination. Since some nodes have more positional power, they are in a position to be more easily affected and also affect other nodes to action $P$.

(v) Standard deviation of degree of $P$ nodes (for "scale-free" networks): The positive effect of the standard deviation of $P$ nodes is the same as the reason in (i) above. A higher standard deviation implies that some $P$ nodes are better connected than others and form hubs. These hubs, due to wider observation span, are more susceptible to action $P$ imitation and thereafter more easily influence a large number of other (connected) nodes.

(vi) Segregation of seed $P$ nodes (for small-world networks): As $P$ nodes are less visible to $R$ nodes, the convergence takes longer.

(vii) Segregation of seed $R$ nodes (for small-world networks): The reason is that a high segregation of $R$ nodes locks many of the $R$ nodes into the use of action $R$.

So far the analysis has been conducted while fixing the size of the network, the average degree of the nodes in the network and the share of the initial action $P$ adopters. ${ }^{17}$ We repeated the analysis in order to assess the effect of marginal changes in one of these parameters. We determine the effect of size by comparing the baseline situation (where size equals 200, average degree equals 6 , and share of initial action $P$ adopters equals 0.20 ) to two alternative situations where only the size of the network changes to 100 and 300 . We find a positive effect for size, i.e. convergence takes longer in a larger population. A similar comparison of the baseline situation to two alternative situations where only the average node degree changes from 6 to 8 reveals that an increase in the average node degree decreases the number of iterations for convergence. The share of initial adopters has the intuitive effect of hastening convergence.

\subsection{Comparing "Scale-Free" and Small-World Networks}

In the preceding subsections, we have investigated the features that promote efficient coordination in "scale-free" and small-world networks, without drawing a comparison between these two network types. In this subsection, we first analyse whether the network type (i.e. "scale-free" or small-world) influences attainment of efficient coordination. We focus on the networks with 200 nodes, average degree equal to 6 and the initial share of action $P$ nodes equal to 0.15 , and we compare the frequency of convergence to the efficient equilibrium in all such "scale-free" networks to small-world networks with relinking probability equal to $0,0.5$ and 1 individually. ${ }^{18}$ The left panel of Fig. 6 shows the distribution of the proportion of times efficient coordination is attained for the "scale-free" networks and for small-world networks with relinking probability equal to $0,0.5$ and 1 . The figure shows that the distribution for "scale-free" networks first order stochastically dominates the distributions for the three small-world networks. ${ }^{19}$ According to Mann-Whitney tests, the distributions are significantly different at the 0.0001 level. These results are robust to marginal changes in size, average degree and share of initial action $P$ adopters.

We also run a regression to see whether the network type has an significant influence on the coordination outcome. The observations come from the "scale-free" and small-world

\footnotetext{
17 The findings are robust to an increase in size or an increase in average degree for "scale-free" networks; for small-world networks, the probability of relinking has a negative effect for more connected networks (average degree equal to 8). This seems to suggest that "hubs" are more important for moderately connected networks but end up being counter-productive for highly connected networks, i.e. higher average connectivity is more crucial than "hubs" for faster convergence.

18 This gives us 10,000 networks each for "scale-free" and small-world networks.

19 For the small-world network, the distribution for the networks with a higher relinking probability first order stochastically dominates the ones with lower relinking probability.
} 


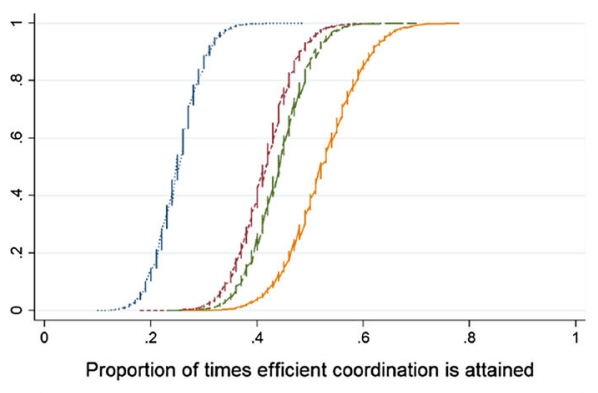

Small-world, relink prob. $=0 \quad \cdots-\cdots-$ Small-world, relink prob. $=0.5$ Small-world, relink prob. $=1 \longrightarrow$ Scale-free

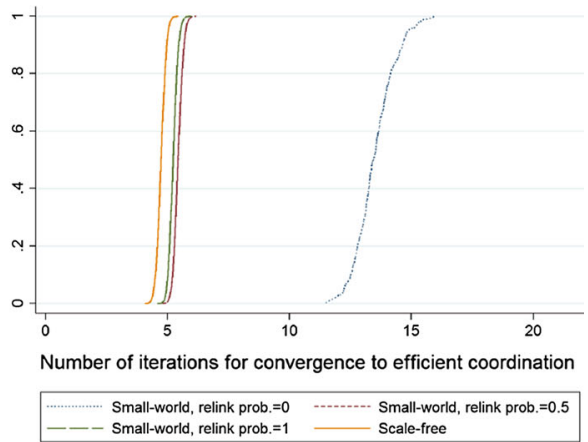

Fig. 6 Distributions of proportion of times efficient coordination is attained $(l e f t)$ and of time taken to attain efficient coordination (right) for small-world and "scale-free" networks

networks with 200 nodes, average degree equal to 6 and the initial share of action $P$ nodes equal to 0.15 , and with relinking probability equal to 1 for small-world networks. ${ }^{20}$ The purpose of this is to see whether there is an effect of the network type after controlling for additional factors such as the degree distribution, the distribution of the power variables or segregation of nodes in the network. The results in column (1) of Table 5 indicate that for a given network size, average degree and share of initial $P$ adopters, it is more likely for a network to converge to efficient coordination if the network is "scale-free" rather than smallworld. ${ }^{21}$ So, there are other differences in the network characteristics between "scale-free" networks and small-world networks beyond those we control for (such as the quintiles of the degree distribution) that influence the coordination process. ${ }^{22}$

Next, we study the impact of network type on the time needed for convergence to the efficient coordination equilibrium. For a given size, average degree and share of initial action $P$ adopters, we examine how the network type affects the time taken to achieve efficient coordination, conditional on it being achieved at least $75 \%$ of the times. To be consistent with the previous subsection, we focus on networks with 200 nodes, average degree equal to 6 and the initial share of action $P$ adopters equal to 0.20 . The right panel of Fig. 6 shows the distribution of the proportion of times efficient coordination is attained for the "scale-free" networks and for small-world networks with relinking probability equal to $0,0.5$ and 1 . The figure shows that the distribution for "scale-free" networks is first order stochastically dominated by the distributions for the three small-world networks. ${ }^{23}$ According to a Mann-Whitney test, these distributions are significantly different at the 0.0001 level. This result is robust to marginal changes in size, average degree and share of initial action $P$ adopters.

\footnotetext{
20 The distribution of proportion of times efficient coordination is attained for small-world networks comes closest to the distribution of "scale-free" networks when the relinking probability for small-world networks equals 1 (see Fig. 6). This drives the choice of the small-world network in the regression.

21 In creating the "network' dummy, small-world networks are assigned a value of 0 and "scale-free" networks are assigned a value of 1 . A positive sign of the network dummy indicated that "scale-free" networks boost efficient coordination after controlling for the factors mentioned earlier.

22 Even though we only show the results of this particular configuration in Table 5, the qualitative effect of the network dummy is same for the other relinking probabilities of the small-world network, and for marginal changes in size and average degree.

23 For the small-world network, the distribution for the networks with a higher relinking probability is first order stochastically dominated by the ones with lower relinking probability.
} 
Table 5 Regression showing the differential effect of network type on efficient coordination [column (1)] and time taken for efficient coordination [column (2)]

(1)

\begin{tabular}{|c|c|c|c|c|}
\hline \multirow[b]{2}{*}{ Variable } & & \\
\hline & Coef. & $\begin{array}{l}\text { Robust } \\
\text { SE. }\end{array}$ & Coef. & $\begin{array}{l}\text { Robust } \\
\text { SE. }\end{array}$ \\
\hline Network $(0=\mathrm{SW} ; 1=\mathrm{SF})$ & $0.0890^{*}$ & $(0.0008)$ & $-0.4739^{*}$ & $(0.0027)$ \\
\hline Degree of $P$ nodes, minimum & $0.0042^{*}$ & $(0.0008)$ & $-0.0157^{*}$ & $(0.0028)$ \\
\hline Degree of $P$ nodes, 75 th percentile & $0.0045^{*}$ & $(0.0006)$ & $0.0230^{*}$ & $(0.0019)$ \\
\hline Power of $P$ nodes, 75 th percentile & $7.4609^{*}$ & $(0.6849)$ & $-8.2095^{*}$ & $(2.3881)$ \\
\hline Segregation (norm.) of $R$ nodes & $-1.5007^{*}$ & $(0.0241)$ & $4.1907^{*}$ & $(0.0542)$ \\
\hline Number of obs. & 20,000 & & 18,592 & \\
\hline R-squared & 0.5521 & & 0.7582 & \\
\hline
\end{tabular}

The stars indicate significance at $0.1 \%$ level

We also run a regression to see whether the time taken for attaining efficient coordination is influenced by the network type. The observations come from the "scale-free" and small-world networks with 200 nodes, average degree equal to 6 and the initial share of action $P$ nodes equal to 0.20 and with relinking probability equal to 1 for small-world networks, conditional on the proportion of achieving efficient coordination being least three-quarters. ${ }^{24}$ The results in column (2) of Table 5 show that it takes more time to reach efficient coordination when the networks are small-world, even after controlling for additional factors such as the degree distribution, the distribution of the power variables and segregation of nodes in the network. ${ }^{25}$

This subsection reveals that the type of network is material for both the likelihood of achieving efficient coordination and the time taken for the same, over and beyond the factors that we have controlled for: efficient coordination is more likely and faster for "scale-free" networks.

\section{Concluding Remarks}

The primary question in coordination games with multiple pure strategy Nash equilibria concerns the possibility of achieving efficient coordination. We consider this question in an environment where individuals are not aware of the entire state of the world but are able to acquire, and base future actions on, information from their (social) network. This is modelled by embedding a coordination game in a set-up where individuals randomly play the coordination game with another individual from the population and imitate the most successful action in their neighbourhood. We identify a few important determinants of the emergent equilibrium behaviour.

The share of initial adopters of the efficient equilibrium action is a crucial determinant. When this share is above (below) a threshold, then the efficient (inefficient) coordination

\footnotetext{
24 As with the likelihood of achieving efficient coordination, the distribution of proportion of times efficient coordination is attained for small-world networks comes closest to the distribution of "scale-free" networks when the relinking probability for small-world networks equals 1 (see Fig. 6).

25 As before, in creating the 'network' dummy, small-world networks are assigned a value of 0 and "scalefree" networks are assigned a value of 1 . A negative sign of the network dummy indicates that it reduces time for efficient coordination to be realised in "scale-free" networks.
} 
obtains, almost irrespective of other factors. This suggests that under the stylised setting here, the network topology does not influence the coordination outcome for a wide range of the share of initial adopters of the efficient equilibrium action. ${ }^{26}$ Only in a small range of moderate levels of the initial share of adopters of the efficient equilibrium, other (network) features play an influential role in the coordination process. Next, for further analysis, we focus on this small range and find that in these situations better connectivity of the initial adopters gives the system a push in the direction of efficient coordination. On the other hand, segregation of the initial adopters impedes efficient coordination in small-world networks. Conditional on achieving efficient coordination, greater average connectivity makes the convergence to this equilibrium faster. Interestingly, some disparity in the level of connectivity (due to the formation of "information hubs") hastens the process of efficient coordination. Factors that delay this include the size of the population and initial segregation of nodes. We also note that given the initial parameters, it is more likely for a "scale-free" network to converge to the efficient coordination outcome.

The results obtained in this paper bring out an interesting contrast between the "mediumrun" and "long-run" outcomes of the process of local imitation and global random interaction. Khan [12] demonstrates that the efficient equilibrium is expected to be the long-run outcome when each node is sufficiently well connected, or when there is no variability in the extent to which nodes are connected, both of which are NSC. The results here indicate that in the medium-run, the variables that arise from the initial distribution of actions (such as initial share of $P$ adopters, factors related to positional power and degree distribution, or segregation indices) are particularly influential when we restrict attention to the cases where the share of initial adopters of the efficient equilibrium action does not entirely dictate the coordination outcome. Thus, depending on the time-horizon, a different set of factors appears to be more informative on the outcome of the process.

While our results indicate that the characteristics of the network may not be the most crucial determinant of the coordination outcome, we speculate that they may be more important when other update rules than the imitate-the-best-neighbour rule are adopted. With the imitate-the-best-neighbour dynamic, observing a single successful instance of the highest payoff results in adoption of the efficient action. In contrast, with a local best-response dynamic (to the strategies in the neighbourhood) or with imitation of the strategy that gives the highest average payoff in the neighbourhood, the coordination process can be expected to be more network dependent, because the best-response or the payoff that gives the highest average payoff depends on the structure of links in the network. In spite of this, it appears reasonable that factors (such as connectivity of the efficient action users or segregation of nodes) identified here as being important will continue to be so. Future research designed to study the informational importance of networks using other updating rules will be instructive in developing a better understanding of coordination on networks.

On a practical note, the findings reported in this paper show that if a social planner wants to implement the efficient outcome, an initiated change in the network structure will not be particularly effective in case the share of nodes (individuals) that are seeded with the desired action is small. Moreover, when this share is beyond a certain threshold, intervention is not needed as efficient coordination can be expected to materialise organically. For intermediate values of this share, intervention can be effective. Our regression results show that for smallworld networks the connectivity (either local or global as measured by positional power) of the well-connected nodes, and the segregation of the nodes that are not seeded with the desired

26 While this is similar in spirit to some papers in the existing literature such as Buskens and Snijders [8] and Tomassini and Pestelacci [21], some of the factors that we identify to be important (such as segregation) have not been studied before. 
action are important variables to consider and to control. While the former objective can be obtained by positioning some nodes as information hubs, the latter objective can be fulfilled by connecting the users of the inefficient equilibrium action to efficient equilibrium action adopters, so that users of the inefficient equilibrium strategy are never too far away from a success story of efficient coordination. Whereas connectivity of highly connected nodes are found to be important for small-world networks, for "scale-free" networks the focus should be more on the subset of nodes that are already playing the desired action. Our regression tree analysis indicates that the segregation of the nodes not playing the desired action is an important predictor for whether the process can organically converge to the efficient outcome.

Acknowledgments We would like to express our thanks to the editor and three anonymous referees for their constructive comments that have helped us making substantial improvements to this article.

Open Access This article is distributed under the terms of the Creative Commons Attribution 4.0 International License (http://creativecommons.org/licenses/by/4.0/), which permits unrestricted use, distribution, and reproduction in any medium, provided you give appropriate credit to the original author(s) and the source, provide a link to the Creative Commons license, and indicate if changes were made.

\section{References}

1. Alós-Ferrer C, Weidenholzer S (2006) Imitation, local interactions, and efficiency. Econ Lett 93(2):163168

2. Alós-Ferrer C, Weidenholzer S (2008) Contagion and efficiency. J Econ Theory 143(1):251-274

3. Antonioni A, Cacault MP, Lalive R, Tomassini M (2013) Coordination on networks: does topology matter? PLoS One 8(2):e55033

4. Ballester PC, Vorsatz M (2014) Random-walk-based segregation measures. Rev Econ Stat 96(3):383-401

5. Barabási A, Albert R (1999) Emergence of scaling in random networks. Science 286(5439):509-512

6. Barabási A, Bonabeau E (2003) Scale-free networks. Sci Am 288(5):60-69

7. Breiman L, Friedman J, Olshen R, Stone C (1984) Classification and regression trees. CRC Press, Boca Raton

8. Buskens V, Snijders C (2008) Effects of network characteristics on reaching the payoff-dominant equilibrium in coordination games: a simulation study. ISCORE discussion paper

9. Davis GF, Yoo M, Baker W (2003) The small world of the American corporate elite: 1982-2001. Strat Organ 1(3):301-326

10. Fleming L, Marx M (2006) Managing creativity in small worlds. Calif Manag Rev 48(4):6-27

11. Kendall MG (1955) Further contributions to the theory of paired comparisons. Biometrics 11(1):43-62

12. Khan A (2014) Coordination under global random interaction and local imitation. Int J Game Theory 43(4):721-745

13. Kogut B, Walker G (2001) The small world of German corporate networks in the global economy. Am Sociol Rev 66(3):317-335

14. Lee IH, Valentinyi A (2000) Noisy contagion without mutation. Rev Econ Stud 67(1):47-56

15. Newman MEJ (2004) Who is the best connected scientist? A study of scientific coauthorship networks. In: Ben-Naim E, Frauenfelder H, Toroczkai Z (eds) Complex networks. Springer, Berlin, pp 337-370

16. Robson A, Vega-Redondo F (1996) Efficient equilibrium selection in evolutionary games with random matching. J Econ Theory 70(1):65-92

17. Roca CP, Sanchez A, Cuesta JA (2012) Individual strategy update and emergence of cooperation in social networks. J Math Sociol 36(1):1-21

18. Roca CP, Cuesta JA, Sanchez A (2009) Promotion of cooperation on networks? The myopic best response case. Eur Phys J B 71(4):587-595

19. Santos FC, Pacheco JM, Lenaerts T (2006) Evolutionary dynamics of social dilemmas in structured populations. Proc Natl Acad Sci 103(9):3490-3494

20. Soramaki K, Bech ML, Arnold J, Glass RJ, Beyeler WE (2007) The topology of interbank payment flows. Phys A 1(1):317-333

21. Tomassini M, Pestelacci E (2010) Evolution of coordination in social networks: a numerical study. Int $\mathbf{J}$ Mod Phys C 21(10):1277-1296 
22. Uzzi B, Amaral LAN, Reed-Tsochas F (2007) Small-world networks and management science research: a review. Eur Manag Rev 4(2):77-91

23. Watts DJ, Strogatz SH (1998) Collective dynamics of 'small-world' networks. Nature 393(6684):440-442

24. Wei T (1952) The algebraic foundation of ranking theory. Ph.D. Thesis, Cambridge University, Cambridge, UK

25. Weidenholzer S (2010) Coordination games and local interactions: a survey of the game theoretic literature. Games 1(4):551-585 